\title{
Situational Awareness of Childhood Immunization in Kenya
}

\author{
Toluwani E. Awoyele ${ }^{\star 2,1}$, Meenal Pore ${ }^{1}$ and Skyler Speakman'
}

${ }^{1}$ IBM, Langata, Kenya; ${ }^{2}$ Carnegie Mellon University, Pittsburgh, PA, USA

\section{Objective}

Large scale surveys has been used extensively to monitor childhood immunization rates. The purpose of this research is to find measurable features that informs the state of immunization in Kenya.

\section{Introduction}

Despite the steady increase in immunization coverage in Kenya, the most recent Kenya Demographic and Health Survey (KDHS) shows that there is still immunization inequality across the country. Nationally, 2 out of every $3(66.67 \%)$ children has been fully immunized but only 2 out of every $5(40 \%)$ children in the North Eastern region were fully vaccinated ${ }^{1}$. There is a need to identify the characteristics of the households with children who are not fully immunized for effective intervention.

\section{Methods}

Kenya Ministry of Health ${ }^{2}$ provides information on required childhood immunization. BCG, DPT 1, 2 \& 3, Polio 1, 2 \& 3, Measles and Vitamin A are required to be given to children across the country at different ages. In this research, a child is considered fully vaccinated if he/she was up to the age of receiving a particular vaccination and had received the vaccination.

26 variables on wealth assets, media exposure and the demographic data of a mother were used as independent variables for building a generalized boosted regression model. The dependent variable was a child being fully vaccinated or not. The receiver operator characteristics (ROC) of the model was examined and the predictor importance of the variables were extracted.

Surveyed households were further clustered into groups using these most important measurable features.

\section{Results}

Initially, 26 variables used was able to classify full immunization at an ROC measure of $64.24 \%$. The predictor importance of these variables can be seen in Figure 1. The region a mother lives in, her highest educational level, the wall material of the house she lives and her frequency of listening to radio were four features with high predictor importance which can be measured without survey data. With these four variables, the model was able to classify at a rate of $63.56 \%$ which is not a significant drop from the initial classification rate.

The 6079 households analyzed were divided into three clusters using these four variables based on Akaike Information Criterion. Information on these clusters can be seen below:

- Low record of being fully up-to-date immunized: The 1325 households in this cluster do not listen to radio at all, have no education, live in houses with natural materials (grass/thatch) and can be found mostly in the North Eastern region of the country. $37.8 \%$ of these households have been fully immunized.

- Intermediate record of being fully up-to-date immunized: The 2817 households in this cluster listen to radio almost every day, have either primary or secondary school education, build their houses with natural wall materials and can be found in Nyanza, Rift Valley and Western part of the country. $52.2 \%$ of these households have been fully immunized.
- High record of being fully up-to-date immunized: The 1933 households in this cluster listen to radio almost every day, have higher, secondary or primary educational levels, build their houses with finished (Cement, Bricks) materials and can be found in Nairobi, Central and Eastern regions of the country. $55.8 \%$ of these households have been fully immunized.

\section{Conclusions}

The region a mother lives, her level of education, her frequency of listening to the radio and house wall materials are informative in predicting whether her child is fully immunized or not. While this gives us some insight on the wealth and demographic characteristics of the mothers whose children are not immunized, we are missing some important information such as health seeking behavior and proximity to healthcare facilities which could influence a mother's decision to immunize her child.

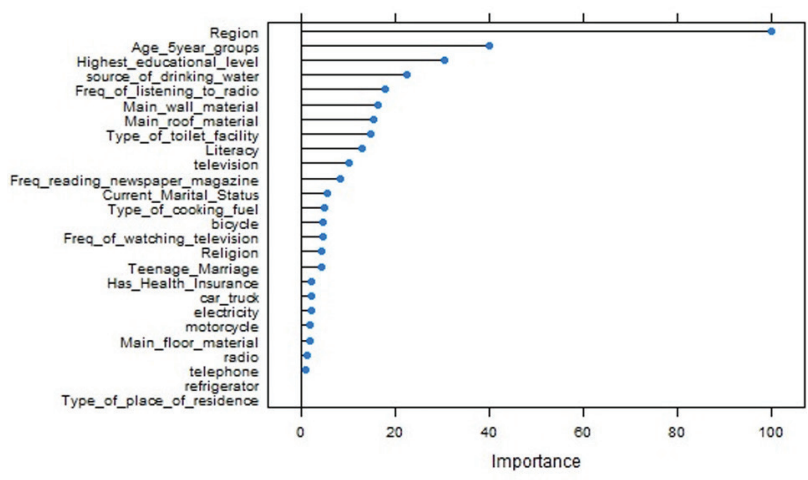

Figure 1

\section{Keywords}

Kenya; Immunization; Clustering

\section{Acknowledgments}

Thanks to United States Agency for International Development (USAID) for providing the dataset used for this analysis.

\section{References}

1. Centers for Disease and Control and Prevention. Kenya joins the world in 'Closing the Immunization Gap'. Global Health - Kenya [Internet]. 2014 April [cited 2015 August 26]; Available from: http://www.cdc. gov/globalhealth/countries/kenya/blog/closing_immunization-_gap. htm

2. Division of Vaccines and Immunization. Multi Year Plan. Republic of Kenya: Ministry of Health; 2010.

\section{*Toluwani E. Awoyele \\ E-mail: tawoyele@andrew.cmu.edu}

